\title{
Microwave photoconductivity of a two-dimensional electron gas due to intra-Landau-level transitions
}

\author{
Christian Joas, Jürgen Dietel, and Felix von Oppen \\ Institut für Theoretische Physik, Freie Universität Berlin, Arnimallee 14, D-14195 Berlin, Germany
}

(Dated: October 23, 2018)

\begin{abstract}
Motivated by a recent experiment, we study the microwave-induced photoconductivity of a twodimensional electron gas arising from intra-Landau-level transitions within a model where the electrons are subject to a unidirectional periodic potential in addition to a weaker impurity potential. With appropriate identifications, our results can be compared to experiment and allow us to explain the sign of the photocurrent, its dependence on magnetic field and microwave frequency as well as the microwave-induced suppression of the Shubnikov-deHaas oscillations.
\end{abstract}

\section{INTRODUCTION}

Recently, novel magnetooscillations of the microwave photoconductivity were discovered in ultra-high-mobility two-dimensional (2d) electron systems in the presence of a weak magnetic field, ${ }^{1,2,3,4,5}$ For sufficiently strong microwave intensity, the longitudinal photoconductivity can become close to zero in certain magnetic field regions, with seemingly activated temperature dependence. Specifically, such so-called "zero-resistance states" occur whenever the microwave frequency $\omega$ is related to the cyclotron frequency $\omega_{c}$ as $\omega=(k+\alpha) \omega_{c}(k=1,2,3 \ldots ; \alpha$ is a constant phase shift).

Several mechanisms have been proposed to account for the photocurrent oscillations, $6,7,8,9,10,11,12,13,14,15,16,17,18$ One mechanism is based on the observation that disorderassisted microwave absorption is accompanied by a realspace displacement which depending on the magnetic field, is preferentially along or against the applied dc electric field 8.9.10.11.12 We refer to this mechanism as displacement mechanism (DP). A second contribution to the photoconductivity arises from the microwave-induced change in the electronic distribution function ${ }^{4.13 .14} \mathrm{It}$ turns out that typically, this distribution-function mechanism (DF) tends to dominate the magnetooscillations of the photoconductivity in realistic samples, ${ }^{14}$ although there are exceptions to this 17 Zero-resistance states are expected to occur once the microwave-induced oscillations become so strong that the microscopic longitudinal conductivity is negative within certain magnetic-field regions, 6.7

In a very recent experiment, Dorozhkin et al. ${ }^{19}$ focused on the regime $\omega \ll \omega_{c}$. Unlike in previous experiments, the microwave irradiation can then only induce intra-LL transitions. According to Dorozhkin et al., a considerable microwave-induced reduction of the diagonal conductivity is observed in this regime, but it appears that no zero-resistance states were found. This overall reduction of the diagonal conductivity is accompanied by a significant suppression of the Shubnikov-deHaas oscillations.

In this paper, we investigate the effect of microwaveinduced intra-Landau-level transitions on the photoconductivity within a model in which the 2DEG is subjected to a unidirectional and static periodic potential.
As shown in Ref. 17, this model allows one to compute the photoconductivity using Fermi's golden rule which, in the appropriate geometry, leads to results which are parametrically consistent with those for disorder-broadened Landau levels. We find that our results are consistent with the principal experimental findings and predict a periodic-potential induced anisotropy of the intra-LL photocurrent.

This paper is organized as follows. In Sec. II we introduce the model and discuss the basic processes which contribute to the photoconductivity for $\omega \ll \omega_{c}$. In Sec. III , we explicitly compute the photoconductivity in this regime, including both the displacement and the distribution-function mechanism. Our results are compared to experiment in Sec. IIID and summarized in Sec. IV]

\section{MODEL AND BASIC PROCESSES}

We consider a two-dimensional electron gas (2DEG) subjected to a perpendicular magnetic field $B$ and a unidirectional, static modulation potential $V(\mathbf{r})=$ $V \cos (Q x)$ of period $a=2 \pi / Q$. We assume that the modulation potential $V(\mathbf{r})$ exceeds the residual disorder potential $U(\mathbf{r})$, whose correlator $W(\mathbf{r})$ falls off isotropically on the scale of the correlation length $\xi$. As appropriate for a high-mobility 2DEG, we assume a smooth disorder potential with correlation length $\xi \gg \lambda_{F} \cdot\left(\lambda_{F}\right.$ denotes the zero-field Fermi wavelength.)

The 2DEG is irradiated by microwaves described by the electric potential

$$
\phi(\mathbf{r}, t)=-\frac{e}{2} \mathbf{r}\left(\mathbf{E}^{*} e^{i \omega t}+\mathbf{E} e^{-i \omega t}\right)=\phi_{+} e^{-i \omega t}+\phi_{-} e^{i \omega t},
$$

where $\phi_{+}=\left[\phi_{-}\right]^{*}=-e \mathbf{E r} / 2$ and $\omega>0$. We first consider linearly polarized microwaves whose polarization vector $\mathbf{E}=E \hat{\mathbf{x}}$ points along the $x$-direction, i.e. parallel to the direction of modulation. Results for more general polarizations will be quoted without derivation.

In the absence of disorder, the Landau level states $|n k\rangle$ in the Landau gauge remain good eigenstates if the amplitude of the periodic modulation is small compared to the LL spacing $\hbar \omega_{c}$. ( $n$ denotes the LL in- 


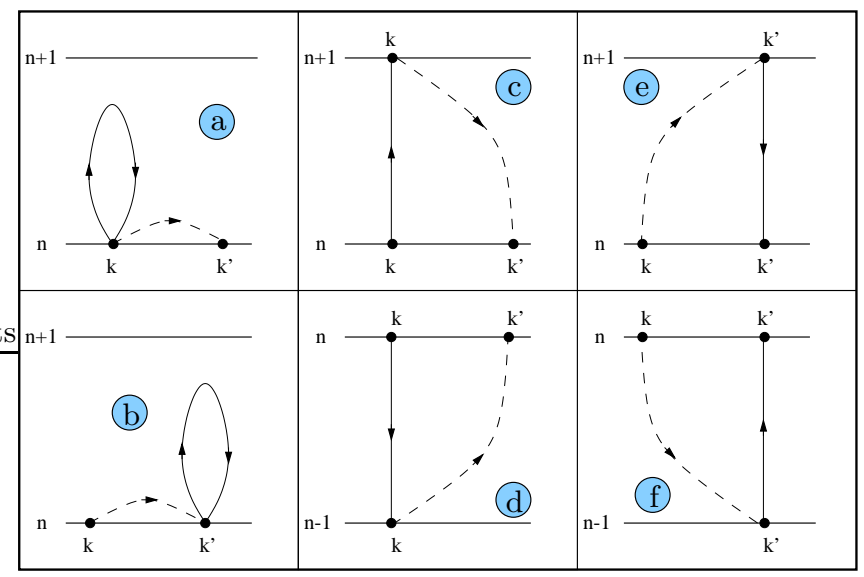

FIG. 1: Relevant processes in the regime $\omega \ll \omega_{c}$. Full lines represent microwave absorption $\left(\phi_{+}\right)$or emission $\left(\phi_{-}\right)$and dashed lines disorder scattering (U). In processes (a) and (b), the intermediate states are in the same (valence) LL as the initial and final states. As shown in the text, these processes dominate the photocurrent. For processes (c)-(f), the LL index of the intermediate states differs by one from the LL index of initial and final states. Their contribution are smaller by a factor $\omega / \omega_{c}$ than (a) and (b).

dex and $k$ the momentum in $y$ direction.) The corresponding eigenenergies are approximately given by $\epsilon_{n k}^{0} \simeq$ $\hbar \omega_{c}\left(n+\frac{1}{2}\right)+V_{n} \cos \left(Q k \ell_{B}^{2}\right)$, where the modulation amplitude $V_{n}$ is given by $V_{n} \simeq V J_{0}\left(q R_{c}\right)$ for large LL index $n$. $\left(\ell_{B}=(\hbar / e B)^{1 / 2}\right.$ is the magnetic length, $R_{c}$ the cyclotron radius, and $J_{n}(z)$ denotes a Bessel function.)

To identify the relevant microwave-induced processes, we discuss the matrix elements of $U$ and $\phi$. For microwaves linearly polarized in the $x$ direction, the matrix elements for absorption and emission are the same and given by

$$
\begin{aligned}
\left\langle n^{\prime} k^{\prime}\left|\phi_{ \pm}\right| n k\right\rangle= & -\frac{e E}{2} k \ell_{B}^{2} \delta_{n, n^{\prime}} \delta_{k, k^{\prime}} \\
& +\frac{e E R_{c}}{4}\left(\delta_{n, n^{\prime}-1}+\delta_{n, n^{\prime}+1}\right) \delta_{k, k^{\prime}} .
\end{aligned}
$$

Thus, microwaves leave the LL index unchanged or couple neighboring LLs. By contrast, the disorder potential has nonzero matrix elements between arbitrary LLs,

$$
\begin{aligned}
& \left|\left\langle n^{\prime} k^{\prime}|U| n k\right\rangle\right|^{2} \\
& \quad \simeq \int \frac{d^{2} q}{(2 \pi)^{2}} \delta_{q_{y}, k^{\prime}-k}\left[J_{\left|n^{\prime}-n\right|}\left(q R_{c}\right)\right]^{2} \tilde{W}(\mathbf{q}) .
\end{aligned}
$$

This expression is valid in the limit of large LL indices $n, n^{\prime} \gg 1$. The dominant microwave-induced processes contributing to the photocurrent arise from the contributions

$$
T_{ \pm}=U G_{0} \phi_{ \pm}+\phi_{ \pm} G_{0} U
$$

to the general $T$-matrix

$$
T=U+\phi+(U+\phi) G_{0}(U+\phi)+\ldots
$$

of the system. 17 Here, $G_{0}$ denotes the retarded Green function of the unperturbed system $(U=\phi=0)$. We refer to $T_{+}\left(T_{-}\right)$as disorder-assisted microwave absorption (emission). $T_{+}$and $T_{-}$can be considered separately, since they contribute incoherently. The matrix elements of $T_{-}$can be shown to equal those of $T_{+}$up to a phase.

We now specialize to the regime $\omega \ll \omega_{c}$ for wellseparated LLs, which is the focus of this paper. In this case, only scattering processes with initial and final state in the same LL are relevant. Disorder-assisted microwave absorption and emission then proceeds via intermediate states either in the same LL (with amplitude $M_{0}$ ) or in neighboring LLs (with amplitude $M_{1}$ ), so that $\left\langle n k^{\prime}\left|T_{+}\right| n k\right\rangle=M_{0}+M_{1}$. These processes are depicted in Fig. 1 (a),(b) and (c)-(f), respectively. We find that the amplitude $M_{1}$ is smaller than $M_{0}$ in the parameter $\omega / \omega_{c}$. Thus, we first turn to the contribution $M_{0}$ which, using Eq. (2), is equal to

$$
\begin{aligned}
M_{0}= & \left\langle n k^{\prime}|U| n k\right\rangle G_{0, n k}\left(\epsilon_{n k}+\omega\right)\left\langle n k\left|\phi_{+}\right| n k\right\rangle \\
& \quad+\left\langle n k^{\prime}\left|\phi_{+}\right| n k^{\prime}\right\rangle G_{0, n k^{\prime}}\left(\epsilon_{n k}\right)\left\langle n k^{\prime}|U| n k\right\rangle \\
= & \frac{e E}{2 \omega}\left(k^{\prime}-k\right) \ell_{B}^{2}\left\langle n k^{\prime}|U| n k\right\rangle .
\end{aligned}
$$

Here we used that $G_{0, n k}\left(\epsilon_{n k}+\omega\right)=\left(\epsilon_{n k}+\omega-\epsilon_{n k}\right)^{-1}=$ $\omega^{-1}$ and $G_{0, n k^{\prime}}\left(\epsilon_{n k}\right)=\left(\epsilon_{n k}-\epsilon_{n k^{\prime}}\right)^{-1}=-\omega^{-1}$. With Eq. (3), we obtain

$$
\left|M_{0}\right|^{2} \simeq\left(\frac{e E}{2 \omega}\right)^{2} \int \frac{d^{2} q}{(2 \pi)^{2}} \delta_{q_{y}, k^{\prime}-k}\left[q_{y} \ell_{B}^{2} J_{0}\left(q R_{c}\right)\right]^{2} \tilde{W}(\mathbf{q})
$$

valid in the limit of high Landau levels.

We now turn to an estimate of the contribution $M_{1}$. The processes depicted in Fig. 1 (c)-(f) lead to

$$
\begin{aligned}
M_{1}= & \left\langle n k^{\prime}|U| n+1 k\right\rangle G_{0, n+1 k}\left(\epsilon_{n k}+\omega\right)\left\langle n+1 k\left|\phi_{+}\right| n k\right\rangle+\left\langle n k^{\prime}|U| n-1 k\right\rangle G_{0, n-1 k}\left(\epsilon_{n k}+\omega\right)\left\langle n-1 k\left|\phi_{+}\right| n k\right\rangle \\
& +\left\langle n k^{\prime}\left|\phi_{+}\right| n-1 k^{\prime}\right\rangle G_{0, n-1 k^{\prime}}\left(\epsilon_{n k}\right)\left\langle n-1 k^{\prime}|U| n k\right\rangle+\left\langle n k^{\prime}\left|\phi_{+}\right| n+1 k^{\prime}\right\rangle G_{0, n+1 k^{\prime}}\left(\epsilon_{n k}\right)\left\langle n+1 k^{\prime}|U| n k\right\rangle \\
= & \frac{e E R_{c}}{4}\left[\frac{\left\langle n k^{\prime}|U| n+1 k\right\rangle}{\omega-\omega_{c}}+\frac{\left\langle n k^{\prime}|U| n-1 k\right\rangle}{\omega+\omega_{c}}-\frac{\left\langle n-1 k^{\prime}|U| n k\right\rangle}{\omega-\omega_{c}}-\frac{\left\langle n+1 k^{\prime}|U| n k\right\rangle}{\omega+\omega_{c}}\right] .
\end{aligned}
$$


At first sight, the ratio $M_{1} / M_{0}$ is of order $\left(R_{c} / q \ell_{B}^{2}\right)\left(\omega / \omega_{c}\right)$. For smooth disorder, $q \sim 1 / \xi$, so that $M_{1} / M_{0} \sim\left(k_{F} \xi\right)\left(\omega / \omega_{c}\right)$, where $k_{F} \xi \gg 1$. This would imply that $M_{1}$ can actually dominate over $M_{0}$. However, this estimate turns out to be too simplistic. The reason is that for $\omega \ll \omega_{c}$, we can write Eq. (8) as

$$
\begin{aligned}
M_{1}= & \frac{e E R_{c}}{4 \omega_{c}}\left\{\left[\left\langle n-1 k^{\prime}|U| n k\right\rangle-\left\langle n k^{\prime}|U| n+1 k\right\rangle\right]\right. \\
& \left.+\left[\left\langle n k^{\prime}|U| n-1 k\right\rangle-\left\langle n+1 k^{\prime}|U| n k\right\rangle\right]\right\}
\end{aligned}
$$

We observe that the square brackets involve differences of matrix elements which differ by a uniform shift by one Landau level. This leads to a partial cancellation which reduces our previous estimate of $M_{1}$ by $q / k_{F} \sim 1 / k_{F} \xi$. As a result, we find that $M_{1} / M_{0} \sim \omega / \omega_{c}$ as claimed above.

\section{PHOTOCURRENT}

\section{A. Mechanisms}

In perturbation theory, disorder-assisted microwave absorption and emission leads to two contributions to the photocurrent. First, it changes the electron momentum from $k$ to $k^{\prime}$, which effectively corresponds to real-space jumps in the $x$ direction of length $\left(k^{\prime}-k\right) \ell_{B}^{2}$. Due to the applied dc electric field, these jumps occur preferentially in a fixed direction. Generalizing the approach of Titeica ${ }^{20}$ to the present situation, this displacement contribution to the longitudinal photocurrent can be expressed as ${ }^{17}$

$$
j_{x}^{\mathrm{DP}}=\frac{\pi e}{L_{x} L_{y}} \sum_{\sigma= \pm} \sum_{n} \sum_{k, k^{\prime}}\left(k^{\prime}-k\right) \ell_{B}^{2}\left|\left\langle n k^{\prime}\left|T_{\sigma}\right| n k\right\rangle\right|^{2}\left[f_{n k}^{0}-f_{n k^{\prime}}^{0}\right] \delta\left(\epsilon_{n k}-\epsilon_{n k^{\prime}}+\sigma \omega\right) .
$$

Here $f_{n k}^{0}$ is the equilibrium electron distribution function and $\epsilon_{n k}=\epsilon_{n k}^{0}-e E_{d c} k \ell_{B}^{2}$ is the Landau level energy including the effect of the dc electric field.

Secondly, the microwaves change the electronic distribution function away from equilibrium. The resulting distribution function contribution to the longitudinal photocurrent is ${ }^{17}$

$$
j_{x}^{\mathrm{DF}}=\frac{\pi e}{L_{x} L_{y}} \sum_{n} \sum_{k, k^{\prime}}\left(k^{\prime}-k\right) \ell_{B}^{2}\left|\left\langle n k^{\prime}|U| n k\right\rangle\right|^{2}\left[\delta f_{n k}-\delta f_{n k^{\prime}}\right] \delta\left(\epsilon_{n k}-\epsilon_{n k^{\prime}}\right),
$$

where $\delta f_{n k}=f_{n k}-f_{n k}^{0}$ is the deviation of the nonequilibrium electron distribution function $f_{n k}$ from the equilibrium distribution $f_{n k}^{0}$. The distribution function $f_{n k}$ can be obtained from the kinetic equation

$$
\frac{\partial f_{n k}}{\partial t}=\left(\frac{\partial f_{n k}}{\partial t}\right)_{\mathrm{dis}}+\left(\frac{\partial f_{n k}}{\partial t}\right)_{\mathrm{mw}}-\frac{f_{\mathrm{nk}}-f_{\mathrm{nk}}^{0}}{\tau_{\mathrm{in}}} .
$$

This kinetic equation includes collision integrals for disorder scattering, $\left(\partial f_{n k} / \partial t\right)_{\mathrm{dis}}=$ $\sum_{n^{\prime} k^{\prime}} 2 \pi\left|\left\langle n^{\prime} k^{\prime}|U| n k\right\rangle\right|^{2}\left[f_{n^{\prime} k^{\prime}}-f_{n k}\right] \delta\left(\epsilon_{n k}-\epsilon_{n^{\prime} k^{\prime}}\right)$, and for disorder-assisted microwave absorption and emission, $\left(\partial f_{n k} / \partial t\right)_{\mathrm{mw}}=\sum_{n^{\prime} k^{\prime}} \sum_{\sigma= \pm} 2 \pi\left|\left\langle n^{\prime} k^{\prime}\left|T_{\sigma}\right| n k\right\rangle\right|^{2}\left[f_{n^{\prime} k^{\prime}}-\right.$ $\left.f_{n k}\right] \delta\left(\epsilon_{n k}-\epsilon_{n^{\prime} k^{\prime}}+\sigma \omega\right)$. These collision integrals involve the electron energies including the effects of the dc electric field. Finally, we include inelastic relaxation within the relaxation-time approximation, with a phenomenological relaxation time $\tau_{\text {in }}$.

\section{B. Longitudinal Photocurrent}

In this section, we compute the photocurrent for dc electric fields applied along the modulation direction. We first turn to the distribution-function mechanism which gives the dominant contribution in the (experimentally relevant) limit of slow inelastic relaxation. The microwave-induced change in the distribution function, as obtained from the kinetic equation (12), equals

$$
\begin{aligned}
\delta f_{N k}=\tau_{\text {in }} & \sum_{k^{\prime}} \sum_{\sigma} 2 \pi\left|\left\langle N k^{\prime}\left|T_{\sigma}\right| N k\right\rangle\right|^{2} \\
& \times\left(f_{N k^{\prime}}^{0}-f_{N k}^{0}\right) \delta\left(\epsilon_{N k}^{0}-\epsilon_{N k^{\prime}}^{0}+\sigma \omega\right),
\end{aligned}
$$

where $N$ denotes the valence Landau level in which the Fermi energy is situated. $\delta f_{n k}$ vanishes for all other Landau levels $n \neq N$. In the limit of $\omega_{c} \gg T \gg V$, the distribution function changes only weakly within the Landau level. Exploiting the $\delta$ function, we can thus write $f_{N k^{\prime}}^{0}-f_{N k}^{0} \simeq-\sigma \beta \omega n_{F}\left(\epsilon_{N k}^{0}\right)\left[1-n_{F}\left(\epsilon_{N k}^{0}\right)\right]$, where $\beta=1 / k_{B} T$ and $n_{F}(\epsilon)$ denotes the Fermi-Dirac distribution. Noting that to leading order $n_{F}\left(\epsilon_{N k}^{0}\right)$ is just the partial filling factor $\nu_{N}^{*}$ of the valence Landau level, we obtain the relation

$$
f_{N k^{\prime}}^{0}-f_{N k}^{0} \simeq-\sigma \beta \omega \nu_{N}^{*}\left(1-\nu_{N}^{*}\right) .
$$

Thus, the change in the distribution function is maximal at the center of the Landau level and falls off to zero towards the Landau-level edges. Inserting the expression 


$$
\delta f_{N k}=-2 \pi \tau_{\text {in }} \beta \omega \nu_{N}^{*}\left(1-\nu_{N}^{*}\right)\left(\frac{e E}{2 \omega}\right)^{2} \sum_{\sigma} \sigma \int \frac{d \mathbf{q}}{(2 \pi)^{2}}\left[q_{y} \ell_{B}^{2} J_{0}\left(q R_{c}\right)\right]^{2} \tilde{W}(\mathbf{q}) \delta\left(\epsilon_{N k}^{0}-\epsilon_{N k+q_{y}}^{0}+\sigma \omega\right) .
$$

The $\mathbf{q}$ integration simplifies significantly in the limit $\lambda_{F} \ll a \ll \ell_{B}^{2} / \xi$, where it factorizes into an average over the $\delta$ function and an integral over the remaining integrand. The average over the $\delta$ function can be expressed through the Landau-level density of states,

$$
\begin{aligned}
& \left\langle\delta\left(\epsilon_{N k}^{0}-\epsilon_{N k+q_{y}}^{0}+\sigma \omega\right)\right\rangle_{k} \\
& \quad=2 \pi \ell_{B}^{2} \nu^{*}\left(\epsilon_{N k}^{0}+\sigma \omega\right) \theta\left(V-\left|\epsilon_{N k}^{0}+\sigma \omega\right|\right) .
\end{aligned}
$$

Here, we introduced the LL density of states $\nu^{*}(\epsilon)=$ $\nu^{*} \tilde{\nu}^{*}(\epsilon)$ where $\nu^{*}=\left(1 / 2 \pi \ell_{B}^{2}\right)\left(1 / \pi V_{N}\right)$ denotes the DOS at the LL center and $\tilde{\nu}^{*}(\epsilon)=1 /\left[1-\left[\left(\epsilon-E_{N}\right) / V_{N}\right]^{2}\right]^{1 / 2}$ a normalized density of states. Exploiting the fact that the integrand of the remaining $q$ integration is cut off by the correlator $\tilde{W}(q)$ at large $q$, we can replace the Bessel function by an asymptotic expression for large argument. In this way, we can relate the integral to the zero-field transport mean free path, defined by

$$
\frac{1}{\tau_{\mathrm{tr}}}=\frac{1}{\pi v_{F}} \int_{0}^{\infty} d q\left(q^{2} / 2 k_{F}^{2}\right) \tilde{W}(q)
$$

This yields for the change in the distribution function

$$
\begin{aligned}
& \delta f_{N k}=-\beta \omega \nu_{N}^{*}\left(1-\nu_{N}^{*}\right)\left(\frac{e E R_{c}}{2 \omega}\right)^{2} \\
& \quad \times \sum_{\sigma} \sigma \frac{\tau_{\text {in }}}{\tau_{\mathrm{tr}}^{*}\left(\epsilon_{N k}^{0}+\sigma \omega\right)} \theta\left(V-\left|\epsilon_{N k}^{0}+\sigma \omega\right|\right) .
\end{aligned}
$$

This expression should be inserted into Eq. (11). Here, we introduced the transport mean free path $\tau_{\mathrm{tr}}^{*}(\epsilon)=$ $\tau_{\operatorname{tr}} \nu / \nu^{*}(\epsilon)$ in the presence of the magnetic field. ( $\nu$ is the DOS in the absence of the $B$-field.) In a smooth random potential, this should be distinguished from the single-particle mean free paths $\tau_{s}$ and $\tau_{s}^{*}(\epsilon)=\tau_{s} \nu / \nu^{*}(\epsilon)$ in the absence and presence of $B$, respectively.

By a sequence of steps very similar to those for the evaluation of $\delta f_{N k}$ just described, we can rewrite Eq. (11) as

$$
\sigma_{x x}^{\mathrm{DF}}=\left[e^{2} \frac{R_{c}^{2}}{2 \tau_{\mathrm{tr}}^{*}} \nu^{*}\right] \frac{2 \pi \ell_{B}^{2}}{L_{x} L_{y}} 2 \pi V \sum_{k}\left(-\frac{\partial \delta f_{N k}}{\partial \epsilon_{N k}^{0}}\right) \tilde{\nu}^{*}\left(\epsilon_{N k}^{0}\right) .
$$

Finally performing the sum over $k$, we obtain the result

$$
\begin{aligned}
\sigma_{x x}^{\mathrm{DF}}= & -2 \beta \omega \nu_{N}^{*}\left(1-\nu_{N}^{*}\right)\left[e^{2} \frac{R_{c}^{2}}{2 \tau_{\mathrm{tr}}^{*}} \nu^{*}\right]\left(\frac{e E R_{c}}{2 \omega}\right)^{2} \frac{\tau_{\text {in }}}{\tau_{\mathrm{tr}}^{*}} \\
& \times B_{1}\left(\omega / 2 V_{N}\right),
\end{aligned}
$$

where

$$
B_{1}\left(\omega / 2 V_{N}\right)=-\frac{\partial}{\partial \omega} \int_{-V}^{V-\omega} d \epsilon\left[\tilde{\nu}^{*}(\epsilon)\right]^{2} \tilde{\nu}^{*}(\epsilon+\omega)
$$

We obtain for this integral

$$
B_{1}(x)=\frac{1}{16} \frac{1-2 x}{\left(x-x^{2}\right)^{3 / 2}} \ln \left(\frac{V_{N}}{\Delta}\right)
$$

Here, $\Delta$ denotes an effective broadening in energy of the LL edge due to disorder or the dc electric field, both of which cut off the logarithmic divergence of the integral.

The sign and frequency dependence of the longitudinal distribution function contribution are therefore determined by the function $-x B_{1}(x)$. This function is plotted in Fig. 2 We find a negative photoconductivity in the frequency range $\omega<V_{N}$. For larger frequencies $V_{N}<\omega<2 V_{N}$, the sign of the photoconductivity changes. This sign change is a specific feature of our model, arising from the singular density of states at the band edge for the static periodic modulation potential. While this sign change is an interesting feature of our model and may be helpful in distinguishing between the displacement and the distribution function mechanism in an appropriate experiment, it is not expected to occur in a more generic situation without a singularity at the Landau level edge. Specifically, one expects a negative photoconductivity for all $\omega<2 V_{N}$ for the case of disorder-broadened Landau levels, relevant to current experiments.

By a similar calculation, starting from Eq. (10), we obtain

$$
\begin{aligned}
\sigma_{x x}^{\mathrm{DP}} \propto & -\beta \omega \nu_{N}^{*}\left(1-\nu_{N}^{*}\right)\left[e^{2} \frac{R_{c}^{2}}{2 \tau_{\mathrm{tr}}^{*}} \nu^{*}\right]\left(\frac{e E R_{c}}{2 \omega}\right)^{2} \frac{\tau_{\mathrm{s}}}{\tau_{\mathrm{tr}}^{*}} \\
& \times A_{1}\left(\omega / 2 V_{N}\right)
\end{aligned}
$$

for the longitudinal displacement photoconductivity. We note in passing that the precise numerical prefactor of $\sigma_{x x}^{\text {DP }}$ depends on the details of the smooth-disorder model. Clearly, this result is parametrically smaller than the distribution-function mechanism by a factor $\tau_{s}^{*} / \tau_{\text {in }}$, where $\tau_{s}^{*}$ denotes the single-particle scattering time in the presence of the magnetic field. The function $A_{1}$ ap- 

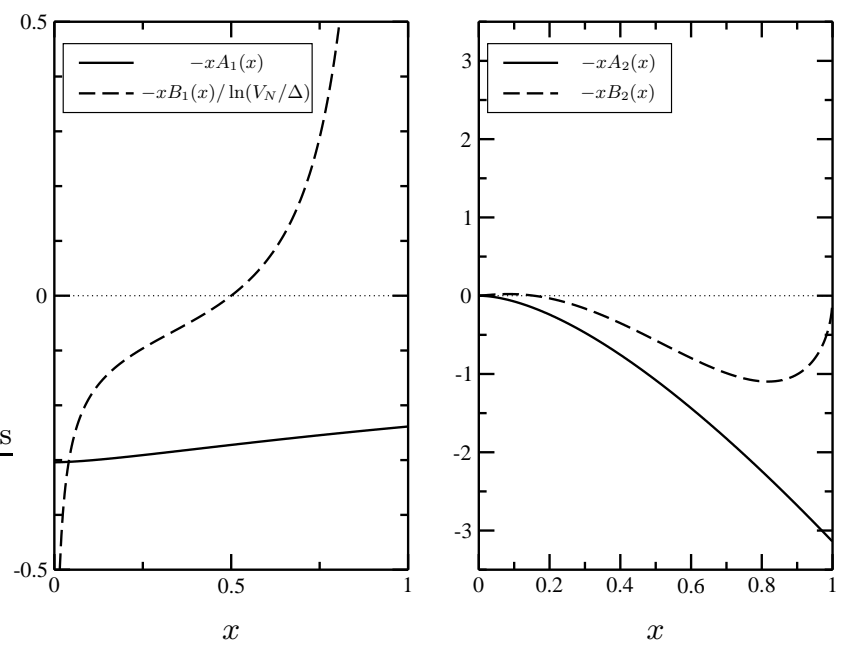

FIG. 2: Sign and frequency dependence of the photocurrent. Left panel: functions $-x A_{1}(x)$ (full line) and $-x B_{1}(x) / \ln \left(V_{N} / \Delta\right)($ dashed) determining the sign and frequency dependence of the displacement and distributionfunction contributions to the longitudinal photoconductivities, respectively (with $x=\omega / 2 V_{N}$ ). Right panel: the corresponding functions $-x A_{2}(x)$ (full line) and $-x B_{2}(x)$ (dashed) for the transverse photocurrent.

pearing in Eq. (23) is given by

$$
\begin{aligned}
A_{1}(x) & =-\frac{3}{2 \pi} \frac{\partial}{\partial x} \frac{\ell_{B}^{2}}{a} \int_{-a / 2 \ell_{B}^{2}}^{a / 2 \ell_{B}^{2}} d q_{y} \frac{1}{\sqrt{\sin ^{2}\left(Q q_{y} \ell_{B}^{2} / 2\right)-x^{2}}} \\
& =-\frac{3}{\pi^{2}} \frac{\partial}{\partial x} K\left(\sqrt{1-x^{2}}\right)
\end{aligned}
$$

and plotted in Fig. 2] Here, $K(x)$ denotes a complete elliptic function.

The dominant distribution-function contribution to the longitudinal photocurrent is independent of the microwave polarization. This is different from the cyclotron-resonance case, 17 where the photocurrent depends on the type of circular polarization ${ }^{21}$

\section{Transverse Photocurrent}

It was shown in Ref. 17 that within our model, the contributions of the displacement and the distributionfunction mechanisms to the transverse photocurrent can be of the same order of magnitude for the cyclotron resonance $\omega \simeq \omega_{c}$. We find the same conclusion to hold for intra-LL transitions.

The methods used in Ref. 17 can be readily extended to intra-LL transitions. The essential new ingredient in computing the distribution-function contribution is a drift term $-e E_{d c}\left(\partial f_{n k} / \partial k\right)$ which enters the RHS of the kinetic equation, Eq. (12). In this way, we arrive at the result

$$
\begin{aligned}
\sigma_{y y}^{\mathrm{DF}}= & -4 \beta \omega \nu_{N}^{*}\left(1-\nu_{N}^{*}\right)\left[e^{2}\left(v_{y}^{2} \tau_{\mathrm{s}}^{*}\right) \tilde{\nu}^{*}\right]\left(\frac{e E R_{c}}{2 \omega}\right)^{2} \frac{\tau_{\mathrm{in}}}{\tau_{\mathrm{tr}}^{*}} \\
& \times B_{2}\left(\omega / 2 V_{N}\right)
\end{aligned}
$$

where $B_{2}\left(\omega / 2 V_{N}\right)$ is given by

$$
B_{2}\left(\omega / 2 V_{N}\right)=-\frac{\partial}{\partial \omega} \int_{-V}^{V-\omega} d \epsilon \frac{1}{\left[\tilde{\nu}^{*}(\epsilon)\right]^{2}} \tilde{\nu}^{*}(\epsilon+\omega)
$$

We obtain for this integral

$$
B_{2}(x)=\left[4 x\left(\arcsin (1-2 x)+\frac{\pi}{2}\right)-4 \sqrt{x-x^{2}}\right] .
$$

Computing the displacement contribution to the transverse photoconductivity requires one to evaluate transition rates between quantum states corresponding to the "meander" equipotential lines in the presence of both static periodic modulation and dc electric field. Following the relevant formalism developed in Ref. 17, we obtain

$$
\begin{aligned}
\sigma_{y y}^{\mathrm{DP}}= & -2 \beta \omega \nu_{N}^{*}\left(1-\nu_{N}^{*}\right)\left[e^{2}\left(v_{y}^{2} \tau_{\mathrm{s}}^{*}\right) \tilde{\nu}^{*}\right]\left(\frac{e E R_{c}}{2 \omega}\right)^{2} \frac{\tau_{\mathrm{in}}}{\tau_{\mathrm{tr}}^{*}} \\
& \times A_{2}\left(\omega / 2 V_{N}\right)\left(\frac{E_{\mathrm{dc}}^{*}}{E_{\mathrm{dc}}}\right)^{2} .
\end{aligned}
$$

The frequency dependence of the photocurrent is described by the function $A_{2}$ given by

$$
\begin{aligned}
A_{2}(x) & =\frac{\pi \ell_{B}^{2}}{a} \int_{-a / 2 \ell_{B}^{2}}^{a / 2 \ell_{B}^{2}} d q_{y} \frac{x}{\sqrt{\sin ^{2}\left(Q q_{y} \ell_{B}^{2} / 2\right)-x^{2}}} \\
& =2 x K\left(\sqrt{1-x^{2}}\right) .
\end{aligned}
$$

A plot of $B_{2}$ and $A_{2}$ is provided in Fig. 2]

Note the singular dependence of the displacement contribution to the transverse photoconductivity on the dc electric field $E_{d c}$. This singularity is cut off for small dc electric fields by inelastic processes when $E_{\mathrm{dc}} \sim E_{\mathrm{dc}}^{*}$, where $E_{\mathrm{dc}}^{*}=B a / 2 \pi \sqrt{\tau_{\mathrm{in} \tau_{s}^{*}}} \frac{17}{}$ For $E_{\mathrm{dc}} \ll E_{\mathrm{dc}}^{*}$, the photoconductivity crosses over to Ohmic behavior, matching with Eq. (28) for $E_{\mathrm{dc}} \sim E_{\mathrm{dc}}^{*} \frac{17}{17}$ This implies that the contributions by displacement and distribution mechanisms are of the same order of magnitude in the transverse case.

We finally remark that both displacement and distribution-function contribution to the intra-LL transverse photocurrent are independent of the type of polarization.

\section{Comparison with experiment}

Strictly speaking, our model is different from the experimental system, due to the assumption of a static periodic potential. However, previous work ${ }^{17}$ shows that the magnitude of the longitudinal photocurrent obtained 
within our model is parametrically identical to that for disorder-broadened Landau levels. Specific features arise within our model due to its anomalous density of states at the LL edge which leads to additional sign changes of the photocurrent.

Keeping these caveats in mind, we compare our results to the experiment of Ref. 19. Our main results relevant to experiment are:

(i) When ignoring effects of the singular density of states at the LL edge, the sign of the photocurrent due to intra-LL transitions is negative, leading to a reduction of the experimentally observed resistivity.

(ii) Comparing the longitudinal photoconductivity in Eq. (20) to the dark conductivity $\sigma_{x x}^{\text {dark }}=$ $e^{2}\left(R_{c}^{2} / 2 \tau_{\mathrm{tr}}^{*}\right) \nu^{*}$, we find that their ratio depends on magnetic field as $\sigma_{x x}^{\mathrm{DF}} / \sigma_{x x}^{\mathrm{dark}} \sim R_{c}^{2} / \tau_{\mathrm{tr}}^{*} \sim 1 / B$ at fixed $\omega$. This magnetic-field dependence actually also holds for inter-LL processes $\underline{17}$

(iii) The amplitude of the photoconductivity due to intra-LL transitions scales as $\sim 1 / \omega$ with the microwave frequency, see Eq. (20).

(iv) Due to the factor $\nu_{N}^{*}\left(1-\nu_{N}^{*}\right)$, the magnitude of the effect is strongest in the LL center and falls off to zero towards the LL edge. We note that this fillingfactor dependence is specific to intra-LL transitions and does not occur for inter-LL transitions near the cyclotron resonance or its harmonics $\underline{17}$

These results are in good agreement with the central experimental observations. (i) explains the sign of the effect. (ii) is in agreement with the observations that over the magnetic-field range where intra-LL processes dominate, the relative microwave-induced suppression of the conductivity decreases as the magnetic field increases (see Fig. 1 of Ref. 19). In addition, this magnetic-field scaling explains why zero-resistance states could not be reached in the regime of intra-LL transitions which occur at higher magnetic fields compared to the cyclotron resonance or its harmonics. (iii) is in accordance with the observation that the microwave-induced reduction of the diagonal resistivity decreases with increasing microwave frequency (see Fig. 2 of Ref. 19). Finally, (iv) implies that the photoconductivity suppresses the Shubnikov-deHaas oscillations, an effect which was very pronounced experimentally.

\section{SUMMARY}

We have studied the microwave photoconductivity of a 2DEG in a perpendicular magnetic field with additional unidirectional static periodic modulation in the regime of intra-LL transitions. We identify the dominant disorderassisted microwave absorption and emission processes for this regime and compute both the longitudinal and transverse photocurrents.

We find that the distribution-function mechanism dominates for the longitudinal photocurrent while both distribution-function and displacement mechanism contribute to the same order to the transverse photocurrent. Except for subdominant contributions, we find that the photoconductivity due to intra-LL processes is polarization independent. The singular density of states of our model near the LL edges leads to interesting sign changes, making the photoconductivity positive in certain frequency ranges. With the exception of these model-specific predictions, our results are in good agreement with experiment. Specifically, we can explain the microwave-induced suppression of the Shubnikov-deHaas oscillations in the regime of intra-LL transitions.

Note: During the completion of this manuscript, we became aware of related work (V. Ryzhii, preprint: cond-mat/0411370 X.L. Lei, S.Y. Liu, preprint: cond-mat/0411717) focusing on intra-LL transitions within different scenarios for the photoconductivity.

\section{Acknowledgments}

This work has been supported by the DFGSchwerpunkt Quanten-Hall-Systeme, and the Junge Akademie.
1 R. G. Mani, J. H. Smet, K. von Klitzing, V. Narayanamurti, W. B. Johnson, and V. Umansky, Nature, 420, 646 (2002).

2 M. A. Zudov, R. R. Du, L. N. Pfeiffer, and K. W. West, Phys. Rev. Lett. 90, 046807 (2003).

3 C. L. Yang, M. A. Zudov, T. A. Knuuttila, R. R. Du, L. N. Pfeiffer and K. W. West, Phys. Rev. Lett. 91, 096803 (2003).

4 S. I. Dorozhkin, JETP Lett. 77, 577 (2003).

${ }^{5}$ R. L. Willett, K. W. West, L. N. Pfeiffer, Bull. Am. Phys. Soc. 48, 459 (2003).
${ }^{6}$ A. V. Andreev, I. L. Aleiner, and A. J. Millis, Phys. Rev. Lett. 91, 056803 (2003).

7 F. S. Bergeret, B. Huckestein, and A. F. Volkov, Phys. Rev. B 67, 241303 (2003).

8 A. Durst, S. Sachdev, N. Read, and S. M. Girvin, Phys. Rev. Lett. 91, 086803 (2003).

9 J. Shi and X. C. Xie, Phys. Rev. Lett. 91, 086801 (2003).

10 X. L. Lei and S. Y. Liu, Phys. Rev. Lett. 91, 226805 (2003).

11 M. G. Vavilov and I. L. Aleiner, Phys. Rev. B, 69, 035303 (2004).

12 V. I. Ryzhii, Fiz. Tverd. Tela 11 , 2577 (1969) [Sov. Phys. 
Solid State 11 , 2078 (1970)]; V. I. Ryzhii, R. A. Suris, and B. S. Shchamkhalova, Fiz. Tekh. Poluprovodn. 20 , 2078 (1986) [Sov. Phys. Semicond. 20 , 1299 (1986)].

13 I. A. Dmitriev, A. D. Mirlin and D. G. Polyakov, Phys. Rev. Lett. 91, 226802 (2003).

14 I. A. Dmitriev, M.G.Vavilov, I. L. Aleiner, A .D. Mirlin and D. G. Polyakov, Phys. Rev. B 70, 165305 (see also preprint cond-mat/03110663).

15 A. A. Koulakov, M. E. Raikh, Phys. Rev. B 68, 115324 (2003).

16 J. P. Robinson, M. P. Kennett, N. R. Cooper, V. I. Fal'ko, Phys. Rev. Lett. 93, 036804 (2004).
17 J. Dietel, L. I. Glazman, F. W. J. Hekking, F. von Oppen, preprint cond-mat/0407298

18 A. Auerbach, I. Finkler, B. I. Halperin, and A. Yacoby, preprint cond-mat/0501170

19 S. I. Dorozhkin, J. H. Smet, V. Umansky, K. von Klitzing, preprint cond-mat/0409228

20 S. Titeica, Ann. Phys. 22, 128 (1935).

21 The displacement photocurrent does exhibit a polarization dependence, but since this contribution is subdominant for the longitudinal photocurrent, we refrain from giving detailed results. 